This is the accepted manuscript of the article, which has been published in Statistical Papers. http://dx.doi.org/10.1007/s00362-017-0943-3

\title{
Some properties of linear sufficiency and the BLUPs in the linear mixed model
}

\author{
S.J. Haslett • X.Q. Liu • \\ A. Markiewicz • S. Puntanen
}

Submitted: 17 February 2017; Revised 26 July 2017

\begin{abstract}
In this paper we consider the linear sufficiency of $\mathbf{F y}$ for $\mathbf{X} \boldsymbol{\beta}$, for $\mathbf{Z u}$ and for $\mathbf{X} \boldsymbol{\beta}+\mathbf{Z u}$, when dealing with the linear mixed model $\mathbf{y}=\mathbf{X} \boldsymbol{\beta}+\mathbf{Z u}+\mathbf{e}$. In particular, we explore the relations between these sufficiency properties. The usual definition of linear sufficiency means, for example, that the BLUE of $\mathbf{X} \boldsymbol{\beta}$ under the original model can be obtained as AFy for some matrix A. Liu et al. (2008) introduced a slightly different definition for the linear sufficiency and we study its relation to the standard definition. We also consider the conditions under which BLUEs and/or BLUPs under one mixed model continue to be BLUEs and/or BLUPs under the other mixed model. In particular, we describe the mutual relations of the conditions. These problems were approached differently by Rong \& Liu (2010) and we will show how their results are related to those obtained by our approach.
\end{abstract}

Keywords Best linear unbiased estimator · best linear unbiased predictor . linear mixed model · linear model · misspecified model.

Mathematics Subject Classification (2000) 62J05 - 62J10

Stephen J. Haslett

Statistical Consulting Unit, The Australian National University, Canberra ACT 2601 Australia

E-mail: stephen.haslett@anu.edu.au

Xu-Qing Liu

College of Science, Nanjing University of Aeronautics and Astronautics, Nanjing 210016 , China, and Faculty of Mathematics and Physics, Huaiyin Institute of Technology, Huai'an 223003, China

E-mail: liuxuqing688@163.com

Augustyn Markiewicz

Department of Mathematical and Statistical Methods, Poznań University of Life Sciences,

Poland

E-mail: amark@up.poznan.pl

Simo Puntanen (author responsible for correspondence)

Faculty of Natural Sciences, FI-33014 University of Tampere, Finland

E-mail: simo.puntanen@uta.fi 


\section{Introduction}

In this paper we consider the general linear model

$$
\mathbf{y}=\mathbf{X} \boldsymbol{\beta}+\varepsilon, \quad \text { or shortly, } \quad \mathscr{M}=\left\{\mathbf{y}, \mathbf{X} \boldsymbol{\beta}, \sigma^{2} \mathbf{V}\right\} .
$$

Here $\mathbf{X}_{n \times p}$ is a known fixed model matrix, the vector $\mathbf{y}$ is an observable $n$-dimensional random vector, $\boldsymbol{\beta}$ is a $p \times 1$ vector of fixed but unknown parameters, and $\varepsilon$ is an unobservable vector of random errors with expectation $\mathrm{E}(\boldsymbol{\varepsilon})=\mathbf{0}$, and covariance matrix $\operatorname{cov}(\boldsymbol{\varepsilon})=\sigma^{2} \mathbf{V}$, where the nonnegative definite matrix $\mathbf{V}$ is known and $\sigma$ is a nonzero positive unknown scalar. For our considerations, however, the scalar $\sigma$ has no role and hence it is omitted.

Let $\mathbf{y}_{*}$ denote a $q \times 1$ unobservable random vector containing new future observations; the words "new" and "future" need not be taken here literally. These new additional observations are assumed to follow the linear model

$$
\mathbf{y}_{*}=\mathbf{X}_{*} \boldsymbol{\beta}+\varepsilon_{*},
$$

where $\mathbf{X}_{*}$ is a known $q \times p$ matrix, $\boldsymbol{\beta}$ is the same vector of unknown parameters as in $\mathscr{M}$, and $\varepsilon_{*}$ is a $q$-dimensional random error vector. The expectation and the covariance matrix are

$$
\mathrm{E}\left(\begin{array}{c}
\mathbf{y} \\
\mathbf{y}_{*}
\end{array}\right)=\left(\begin{array}{c}
\mathbf{X} \boldsymbol{\beta} \\
\mathbf{X}_{*} \boldsymbol{\beta}
\end{array}\right)=\left(\begin{array}{c}
\mathbf{X} \\
\mathbf{X}_{*}
\end{array}\right) \boldsymbol{\beta}, \quad \operatorname{cov}\left(\begin{array}{c}
\mathbf{y} \\
\mathbf{y}_{*}
\end{array}\right)=\left(\begin{array}{cc}
\mathbf{V} & \mathbf{V}_{12} \\
\mathbf{V}_{21} & \mathbf{V}_{22}
\end{array}\right)=\boldsymbol{\Gamma},
$$

where the covariance matrix $\boldsymbol{\Gamma}$ is assumed to be known. For brevity, we denote this setup as

$$
\mathscr{M}_{*}=\left\{\left(\begin{array}{c}
\mathbf{y} \\
\mathbf{y}_{*}
\end{array}\right),\left(\begin{array}{c}
\mathbf{X} \\
\mathbf{X}_{*}
\end{array}\right) \boldsymbol{\beta},\left(\begin{array}{cc}
\mathbf{V} & \mathbf{V}_{12} \\
\mathbf{V}_{21} & \mathbf{V}_{22}
\end{array}\right)\right\},
$$

and call $\mathscr{M}_{*}$ as the "linear model with new observations".

By the linear mixed model we mean

$$
\mathbf{y}=\mathbf{X} \boldsymbol{\beta}+\mathbf{Z u}+\mathbf{e}, \quad \text { or shortly, } \mathscr{L}=\{\mathbf{y}, \mathbf{X} \boldsymbol{\beta}+\mathbf{Z} \mathbf{u}, \mathbf{D}, \mathbf{R}, \mathbf{S}\},
$$

where $\mathbf{Z}_{n \times q}$ is a known matrix, $\mathbf{y}, \mathbf{X}$, and $\boldsymbol{\beta}$ are as in $\mathscr{M}$ but $\mathbf{u}$ is an unobservable $q$-dimensional random effect with $\mathrm{E}(\mathbf{u})=\mathbf{0}, \operatorname{cov}(\mathbf{u})=\mathbf{D}$, and $\mathbf{e}$ is a random error vector with $\mathrm{E}(\mathbf{e})=\mathbf{0}, \operatorname{cov}(\mathbf{e})=\mathbf{R}, \operatorname{cov}(\mathbf{e}, \mathbf{u})=\mathbf{S}$. In this situation

$$
\operatorname{cov}(\mathbf{y})=\operatorname{cov}(\mathbf{Z u}+\mathbf{e})=\mathbf{Z D Z} \mathbf{Z}^{\prime}+\mathbf{R}+\mathbf{Z S}^{\prime}+\mathbf{S Z}^{\prime}=\boldsymbol{\Sigma} .
$$

As for notation, let $\mathbb{R}^{m \times n}$ denote the set of $m \times n$ real matrices. The symbols $\mathbf{A}^{\prime}, \mathbf{A}^{-}, \mathbf{A}^{+}, \mathscr{C}(\mathbf{A})$, and $\mathscr{C}(\mathbf{A})^{\perp}$, denote, respectively, the transpose, a generalized inverse, the Moore-Penrose inverse, the column space, and the orthogonal complement of the column space of the matrix $\mathbf{A}$. By $(\mathbf{A}: \mathbf{B})$ we denote the partitioned matrix with $\mathbf{A}_{m \times n}$ and $\mathbf{B}_{m \times k}$ as submatrices. By $\mathbf{A}^{\perp}$ we denote any matrix satisfying $\mathscr{C}\left(\mathbf{A}^{\perp}\right)=\mathscr{C}(\mathbf{A})^{\perp}$. Furthermore, we will write $\mathbf{P}_{\mathbf{A}}=\mathbf{A A}^{+}=\mathbf{A}\left(\mathbf{A}^{\prime} \mathbf{A}\right)^{-} \mathbf{A}^{\prime}$ to denote the orthogonal projector (with respect 
to the standard inner product) onto $\mathscr{C}(\mathbf{A})$, and $\mathbf{Q}_{\mathbf{A}}=\mathbf{I}-\mathbf{P}_{\mathbf{A}}$. In particular, we denote $\mathbf{M}=\mathbf{I}_{n}-\mathbf{P}_{\mathbf{X}}$. One choice for $\mathbf{X}^{\perp}$ is of course $\mathbf{M}$.

The linear estimator $\mathbf{G y}$ is the best linear unbiased estimator, BLUE, of $\mathbf{X} \boldsymbol{\beta}$ whenever $\mathbf{G y}$ is unbiased and it has the smallest covariance matrix (in the Löwner sense) among all linear unbiased estimators of $\mathbf{X} \boldsymbol{\beta}$. The following lemma characterises the BLUE; see, e.g., Drygas (1970, p. 55), Rao (1973 p. 282).

Lemma 1 Consider the general linear model $\mathscr{M}=\{\mathbf{y}, \mathbf{X} \boldsymbol{\beta}, \mathbf{V}\}$. Then the estimator $\mathbf{G y}$ is the BLUE for $\mathbf{X} \boldsymbol{\beta}$ if and only if $\mathbf{G}$ satisfies the equation

$$
\mathbf{G}\left(\mathbf{X}: \mathbf{V X}^{\perp}\right)=(\mathbf{X}: \mathbf{0})
$$

The corresponding condition for Ay to be the BLUE of an estimable parametric function $\mathbf{K} \boldsymbol{\beta}$, i.e., $\mathscr{C}\left(\mathbf{K}^{\prime}\right) \subset \mathscr{C}\left(\mathbf{X}^{\prime}\right)$, is

$$
\mathbf{A}\left(\mathbf{X}: \mathbf{V} \mathbf{X}^{\perp}\right)=(\mathbf{K}: \mathbf{0}) .
$$

One well-known solution for $\mathbf{G}$ in (1.7) (which is always solvable) is

$$
\mathbf{P}_{\mathbf{X} ; \mathbf{W}^{-}}=\mathbf{X}\left(\mathbf{X}^{\prime} \mathbf{W}^{-} \mathbf{X}\right)^{-} \mathbf{X}^{\prime} \mathbf{W}^{-}
$$

where $\mathbf{W}$ belongs to the set of nonnegative definite matrices defined as

$$
\mathcal{W}_{\mathbf{V}}=\left\{\mathbf{W} \in \mathbb{R}^{n \times n}: \mathbf{W}=\mathbf{V}+\mathbf{X} \mathbf{U U}^{\prime} \mathbf{X}^{\prime}, \mathscr{C}(\mathbf{W})=\mathscr{C}(\mathbf{X}: \mathbf{V})\right\}
$$

It is not necessary, see Kala et al. $(2017, \S 4)$, to restrict the class $\mathcal{W}_{\mathbf{V}}$ to contain only nonnegative definite matrices, that is, we could consider matrices of the type $\mathbf{W}=\mathbf{V}+\mathbf{X} \mathbf{N} \mathbf{X}^{\prime}$, where $\mathbf{N}$ is such that $\mathscr{C}(\mathbf{W})=\mathscr{C}(\mathbf{X}: \mathbf{V})$. However, for our purposes it is notationally a bit simpler to use $\mathcal{W}_{\mathbf{V}}$ as defined in $(1.10)$.

The random vector $\mathbf{y}_{*}$ is said to be predictable if it has a linear unbiased predictor $\mathbf{C y}$, say, i.e., there exists a matrix $\mathbf{C}$ such that $\mathrm{E}\left(\mathbf{y}_{*}-\mathbf{C y}\right)=\mathbf{0}$ for all $\boldsymbol{\beta} \in \mathbb{R}^{p}$. This is equivalent to $\mathbf{X}_{*}^{\prime}=\mathbf{X}^{\prime} \mathbf{C}^{\prime}$, i.e., to the inclusion $\mathscr{C}\left(\mathbf{X}_{*}^{\prime}\right) \subset \mathscr{C}\left(\mathbf{X}^{\prime}\right)$ which is the condition for the estimability of $\mathbf{X}_{*} \boldsymbol{\beta}$ under $\mathscr{M}$. Now a linear unbiased predictor By is the best linear unbiased predictor, BLUP, for $\mathbf{y}_{*}$, if the Löwner ordering

$$
\operatorname{cov}\left(\mathbf{y}_{*}-\mathbf{B y}\right) \leq_{\mathrm{L}} \operatorname{cov}\left(\mathbf{y}_{*}-\mathbf{N y}\right)
$$

holds for all $\mathbf{N}$ such that $\mathbf{N y}$ is an linear unbiased predictor for $\mathbf{y}_{*}$.

The following lemma characterises the BLUP; for the proof, see, e.g., Christensen (2011, p. 294), and Isotalo \& Puntanen (2006, p. 1015).

Lemma 2 Consider the linear model $\mathscr{M}_{*}$ (with new observations) and let $\mathbf{y}_{*}$ be predictable. Then the linear predictor $\mathbf{B y}$ is the best linear unbiased predictor, BLUP, for $\mathbf{y}_{*}$ if and only if $\mathbf{B}$ satisfies the equation

$$
\mathbf{B}\left(\mathbf{X}: \mathbf{V} \mathbf{X}^{\perp}\right)=\left(\mathbf{X}_{*}: \mathbf{V}_{21} \mathbf{X}^{\perp}\right)=\left[\mathbf{X}_{*}: \operatorname{cov}\left(\mathbf{y}_{*}, \mathbf{y}\right) \mathbf{X}^{\perp}\right]
$$


Denoting $\mathbf{X}_{*}=\mathbf{C X}$ (indicating that $\mathbf{y}_{*}$ is predictable) and $\mathbf{T}=\mathbf{P}_{\mathbf{X} ; \mathbf{W}^{-}}$, we have, for example, the following representations for the BLUP of $\mathbf{y}_{*}$; see Haslett et al. (2014, Th. 2):

$$
\begin{aligned}
\operatorname{BLUP}\left(\mathbf{y}_{*} \mid \mathscr{L}\right) & =\mathbf{X}_{*}\left(\mathbf{X}^{\prime} \mathbf{W}^{-} \mathbf{X}\right)^{-} \mathbf{X}^{\prime} \mathbf{W}^{-} \mathbf{y}+\mathbf{V}_{21} \mathbf{V}^{-}\left(\mathbf{I}_{n}-\mathbf{T}\right) \mathbf{y} \\
& =\mathbf{C} \mathbf{T} \mathbf{y}+\mathbf{V}_{21} \mathbf{V}^{-}\left(\mathbf{I}_{n}-\mathbf{T}\right) \mathbf{y} \\
& =\mathbf{C T y}+\mathbf{V}_{21} \mathbf{W}^{-}\left(\mathbf{I}_{n}-\mathbf{T}\right) \mathbf{y} \\
& =\mathbf{C T y}+\mathbf{V}_{21} \mathbf{M}(\mathbf{M V M})^{-} \mathbf{M y}
\end{aligned}
$$

The general representation for the BLUP of $\mathbf{y}_{*}$ under $\mathscr{M}_{*}$ can be written, for example, as $\mathbf{B}_{0} \mathbf{y}$, where

$$
\mathbf{B}_{0}=\mathbf{C T}+\mathbf{V}_{21} \mathbf{V}^{-}\left(\mathbf{I}_{n}-\mathbf{T}\right)+\mathbf{H Q} \mathbf{W},
$$

with $\mathbf{H} \in \mathbb{R}^{n \times n}$ being free to vary. In this context it may be worth emphasizing that the realised value of $\mathbf{B}_{0} \mathbf{y}$ is invariant with respect to the choice of the generalized inverses involved; this is so in view of Rao \& Mitra (1971, Lemma 2.2.4) and the assumed consistency of the model indicating that $\mathbf{y} \in \mathscr{C}(\mathbf{W})$ with probability 1 .

Let us formally define the concept of linear sufficiency as done by Baksalary \& Kala (1981, 1986) and Drygas (1983).

Definition 1 A linear statistic $\mathbf{F y}$, where $\mathbf{F} \in \mathbb{R}^{f \times n}$, is called linearly sufficient for $\mathbf{X} \boldsymbol{\beta}$ under the model $\mathscr{M}=\{\mathbf{y}, \mathbf{X} \boldsymbol{\beta}, \mathbf{V}\}$, if there exists a matrix $\mathbf{A} \in \mathbb{R}^{n \times f}$ such that AFy is the BLUE for $\mathbf{X} \boldsymbol{\beta}$. Correspondingly, Fy is linearly sufficient for estimable $\mathbf{K} \boldsymbol{\beta}$, where $\mathbf{K} \in \mathbb{R}^{k \times p}$, if there exists a matrix $\mathbf{A} \in \mathbb{R}^{k \times f}$ such that $\mathbf{A F y}$ is the BLUE for $\mathbf{K} \boldsymbol{\beta}$; that is, there exists a matrix A such that

$$
\mathbf{A F}(\mathbf{X}: \mathbf{V M})=(\mathbf{K}: \mathbf{0}) .
$$

The concept of linear prediction sufficiency is defined analogically:

Definition 2 Let $\mathbf{y}_{*}=\mathbf{X}_{*} \boldsymbol{\beta}+\varepsilon_{*}$ be predictable under the model $\mathscr{M}_{*}$, i.e., $\mathscr{C}\left(\mathbf{X}_{*}^{\prime}\right) \subset \mathscr{C}\left(\mathbf{X}^{\prime}\right)$. Then $\mathbf{F y}$ is called linearly prediction sufficient for $\mathbf{y}_{*}$ if there exists a matrix $\mathbf{A}$ such that $\mathbf{A F y}$ is the BLUP for $\mathbf{y}_{*}$; that is, there exists a matrix A such that

$$
\mathbf{A F}(\mathbf{X}: \mathbf{V M})=\left(\mathbf{X}_{*}: \mathbf{V}_{21} \mathbf{M}\right)=\left[\mathbf{X}_{*}: \operatorname{cov}\left(\mathbf{y}_{*}, \mathbf{y}\right) \mathbf{M}\right] .
$$

We will sometimes use the phrase "BLUE-sufficient" and the notation $\mathbf{F y} \in$ $\mathcal{S}(\mathbf{K} \boldsymbol{\beta})$. Correspondingly, we may use the term "BLUP-sufficient" and the notation $\mathbf{F y} \in \mathcal{S}\left(\mathbf{y}_{*}\right)$. However, the division into BLUE-sufficiency vs. BLUPsufficiency is not necessary and we can simply refer to linear sufficiency of $\mathbf{F y}$ with respect to $\mathbf{K} \boldsymbol{\beta}$ or $\mathbf{y}_{*}$. Thus we have, for example,

$$
\begin{aligned}
\mathcal{S}(\mathbf{K} \boldsymbol{\beta}) & =\{\mathbf{F y}: \mathbf{A F}(\mathbf{X}: \mathbf{V M})=(\mathbf{K}: \mathbf{0}) \text { for some } \mathbf{A}\}, \\
\mathcal{S}\left(\mathbf{y}_{*}\right) & =\left\{\mathbf{F y}: \mathbf{A F}(\mathbf{X}: \mathbf{V M})=\left(\mathbf{X}_{*}: \mathbf{V}_{21} \mathbf{M}\right) \text { for some } \mathbf{A}\right\} .
\end{aligned}
$$


As Kala et al. (2017, Remark 2) point out, the notation of the type 1.17) is merely symbolic and it is not meant to refer to a set containing only one element which is a single fixed vector resulting from transformation of an observed vector $\mathbf{y}$, or is a single random vector variable being a specific linear transformation of the random vector $\mathbf{y}$. We are, of course, actually interested in the set of matrices $\mathbf{F}$ satisfying a certain property.

For the following Lemma 3, see, e.g., Baksalary \& Kala $(1981,1986)$, Drygas (1983), Tian \& Puntanen (2009, Th. 2.8), and Kala et al. (2017, Th. 2).

Lemma 3 The statistic $\mathbf{F y}$ is BLUE-sufficient for $\mathbf{X} \boldsymbol{\beta}$ under the model $\mathscr{M}=$ $\{\mathbf{y}, \mathbf{X} \boldsymbol{\beta}, \mathbf{V}\}$ if and only if any of the following equivalent statements holds:

(a) $\mathscr{C}\left(\begin{array}{c}\mathbf{X}^{\prime} \\ \mathbf{0}\end{array}\right) \subset \mathscr{C}\left(\begin{array}{c}\mathbf{X}^{\prime} \mathbf{F}^{\prime} \\ \mathbf{M V \mathbf { F } ^ { \prime }}\end{array}\right)$,

(b) $\mathscr{C}(\mathbf{X}) \subset \mathscr{C}\left(\mathbf{W F}^{\prime}\right)$, where $\mathbf{W} \in \mathcal{W}_{\mathbf{V}}$ and $\mathcal{W}_{\mathbf{V}}$ is defined as in 1.10,

(c) $\mathscr{C}\left(\mathbf{X}^{\prime} \mathbf{F}^{\prime}\right)=\mathscr{C}\left(\mathbf{X}^{\prime}\right)$ and $\mathscr{C}(\mathbf{F X}) \cap \mathscr{C}\left(\mathbf{F} \mathbf{V} \mathbf{X}^{\perp}\right)=\{\mathbf{0}\}$.

Let $\mathbf{K} \boldsymbol{\beta}$ be estimable under $\mathscr{M}$. Then $\mathbf{F y}$ is BLUE-sufficient for $\mathbf{K} \boldsymbol{\beta}$ if and only if

(d) $\mathscr{C}\left(\begin{array}{c}\mathbf{K}^{\prime} \\ \mathbf{0}\end{array}\right) \subset \mathscr{C}\left(\begin{array}{c}\mathbf{X}^{\prime} \mathbf{F}^{\prime} \\ \mathbf{M V F}\end{array}\right)$.

Suppose that $\mathbf{y}_{*}=\mathbf{X}_{*} \boldsymbol{\beta}+\boldsymbol{\varepsilon}_{*}$ is predictable under $\mathscr{M}_{*}$. Then $\mathbf{F y}$ is BLUPsufficient for $\mathbf{y}_{*}$ if and only if

(e) $\mathscr{C}\left(\begin{array}{c}\mathbf{X}_{*}^{\prime} \\ \mathbf{M} \mathbf{V}_{12}\end{array}\right) \subset \mathscr{C}\left(\begin{array}{c}\mathbf{X}^{\prime} \mathbf{F}^{\prime} \\ \mathbf{M} \mathbf{V} \mathbf{F}^{\prime}\end{array}\right)$.

The structure of the paper is as follows. In Section 2 we consider the linear sufficiency of $\mathbf{F y}$ for $\mathbf{X} \boldsymbol{\beta}$, for $\mathbf{Z u}$ and for $\mathbf{X} \boldsymbol{\beta}+\mathbf{Z u}$, when dealing with the linear mixed model $\mathbf{y}=\mathbf{X} \boldsymbol{\beta}+\mathbf{Z u}+\mathbf{e}$. In particular, we explore the relations between these sufficiency properties. Liu et al. (2008) introduced a slightly different definition for the linear sufficiency and we study its relation to the standard definition. In Section 3 we consider the conditions under which BLUEs and/or BLUPs under one mixed model continue to be BLUEs and/or BLUPs under the other mixed model. In particular, we describe the mutual relations of the conditions. These problems were approached differently by Rong \& Liu (2010) and we will show how their results are related to those obtained by our approach.

\section{Linear sufficiency in a linear mixed model}

Consider the linear mixed model

$$
\mathbf{y}=\mathbf{X} \boldsymbol{\beta}+\mathbf{Z u}+\mathbf{e}, \quad \text { denoted as } \mathscr{L}=\{\mathbf{y}, \mathbf{X} \boldsymbol{\beta}+\mathbf{Z} \mathbf{u}, \mathbf{D}, \mathbf{R}, \mathbf{S}\},
$$

where $\mathbf{X}_{n \times p}$ and $\mathbf{Z}_{n \times q}$ are known matrices, $\boldsymbol{\beta} \in \mathbb{R}^{p}$ is a vector of unknown fixed effects, $\mathbf{u}$ is an unobservable vector ( $q$ elements) of random effects with 
$\mathrm{E}(\mathbf{u})=\mathbf{0}, \operatorname{cov}(\mathbf{u})=\mathbf{D}_{q \times q}, \operatorname{cov}(\mathbf{e}, \mathbf{u})=\mathbf{S}_{n \times q}$, and $\mathrm{E}(\mathbf{e})=\mathbf{0}, \operatorname{cov}(\mathbf{e})=\mathbf{R}_{n \times n}$. In this situation

$$
\operatorname{cov}\left(\begin{array}{l}
\mathbf{e} \\
\mathbf{u}
\end{array}\right)=\left(\begin{array}{ll}
\mathbf{R} & \mathbf{S} \\
\mathbf{S}^{\prime} & \mathbf{D}
\end{array}\right), \quad \operatorname{cov}\left(\begin{array}{l}
\mathbf{y} \\
\mathbf{u}
\end{array}\right)=\left(\begin{array}{cc}
\boldsymbol{\Sigma} & \mathbf{Z D}+\mathbf{S} \\
(\mathbf{Z D}+\mathbf{S})^{\prime} & \mathbf{D}
\end{array}\right),
$$

and $\operatorname{cov}(\mathbf{y})=\mathbf{Z D Z} \mathbf{Z}^{\prime}+\mathbf{R}+\mathbf{Z S}^{\prime}+\mathbf{S Z}^{\prime}=\mathbf{\Sigma}$.

The mixed model can be expressed as a version of the model with "new observations", the new observations being now in $\mathbf{u}$ :

$$
\left\{\left(\begin{array}{l}
\mathbf{y} \\
\mathbf{u}
\end{array}\right),\left(\begin{array}{c}
\mathbf{X} \\
\mathbf{0}
\end{array}\right) \boldsymbol{\beta},\left(\begin{array}{cc}
\mathbf{\Sigma} & \mathbf{Z D}+\mathbf{S} \\
\mathbf{D Z} \mathbf{Z}^{\prime}+\mathbf{S}^{\prime} & \mathbf{D}
\end{array}\right)\right\} .
$$

Corresponding to (1.1) and $(1.2)$, we have

$$
\begin{aligned}
& \mathbf{y}=\mathbf{X} \boldsymbol{\beta}+\varepsilon, \quad \operatorname{cov}(\varepsilon)=\boldsymbol{\Sigma}, \varepsilon=\mathbf{Z u}+\mathbf{e}, \\
& \mathbf{u}=\boldsymbol{\varepsilon}_{*}, \quad \operatorname{cov}\left(\boldsymbol{\varepsilon}_{*}\right)=\mathbf{D}, \operatorname{cov}\left(\varepsilon, \boldsymbol{\varepsilon}_{*}\right)=\mathbf{Z D}+\mathbf{S} .
\end{aligned}
$$

Moreover, choosing the "new observations" as $\mathbf{g}=\mathbf{X} \boldsymbol{\beta}+\mathbf{Z u}$, we get

$$
\left\{\left(\begin{array}{l}
\mathbf{y} \\
\mathbf{g}
\end{array}\right),\left(\begin{array}{l}
\mathbf{X} \\
\mathbf{X}
\end{array}\right) \boldsymbol{\beta},\left(\begin{array}{cc}
\mathbf{\Sigma} & (\mathbf{Z D}+\mathbf{S}) \mathbf{Z}^{\prime} \\
\mathbf{Z}\left(\mathbf{D} \mathbf{Z}^{\prime}+\mathbf{S}^{\prime}\right) & \mathbf{Z D Z} \mathbf{Z}^{\prime}
\end{array}\right)\right\} .
$$

Thus, see, e.g., Haslett et al. (2015), under the mixed model $\mathscr{L}$ the following statements hold:

(a) $\mathbf{A y}$ is the BLUE for $\mathbf{X} \boldsymbol{\beta}$ if and only if

$$
\mathbf{A}(\mathbf{X}: \mathbf{\Sigma M})=(\mathbf{X}: \mathbf{0}) .
$$

It is worth noting that the result 2.6) follows at once from 1.7 by observing that 2.5 includes a standard submodel $\{\mathbf{y}, \mathbf{X} \boldsymbol{\beta}, \boldsymbol{\Sigma}\}$.

(b) $\mathbf{B}_{1} \mathbf{y}$ is the BLUP for $\mathbf{u}$ if and only if

$$
\mathbf{B}_{1}(\mathbf{X}: \mathbf{\Sigma} \mathbf{M})=\left[\mathbf{0}:\left(\mathbf{D Z} \mathbf{Z}^{\prime}+\mathbf{S}^{\prime}\right) \mathbf{M}\right]=[\mathbf{0}: \operatorname{cov}(\mathbf{u}, \mathbf{y}) \mathbf{M}] .
$$

(c) $\mathbf{B}_{2} \mathbf{y}$ is the BLUP for $\mathbf{g}=\mathbf{X} \boldsymbol{\beta}+\mathbf{Z u}$ if and only if

$$
\mathbf{B}_{2}(\mathbf{X}: \mathbf{\Sigma M})=\left[\mathbf{X}: \mathbf{Z}\left(\mathbf{D} \mathbf{Z}^{\prime}+\mathbf{S}^{\prime}\right) \mathbf{M}\right]=[\mathbf{X}: \operatorname{cov}(\mathbf{g}, \mathbf{y}) \mathbf{M}] .
$$

(d) $\mathbf{B}_{3} \mathbf{y}$ is the BLUP for $\mathbf{Z u}$ if and only if

$$
\mathbf{B}_{3}(\mathbf{X}: \mathbf{\Sigma} \mathbf{M})=\left[\mathbf{0}: \mathbf{Z}\left(\mathbf{D} \mathbf{Z}^{\prime}+\mathbf{S}^{\prime}\right) \mathbf{M}\right]=[\mathbf{0}: \operatorname{cov}(\mathbf{Z u}, \mathbf{y}) \mathbf{M}] .
$$

(e) $\mathbf{B}_{4} \mathbf{y}$ is the BLUP for $\boldsymbol{\eta}=\mathbf{K} \boldsymbol{\beta}+\mathbf{L u}$ if and only if

$$
\mathbf{B}_{4}(\mathbf{X}: \mathbf{\Sigma M})=\left[\mathbf{K}: \mathbf{L}\left(\mathbf{D Z} \mathbf{Z}^{\prime}+\mathbf{S}^{\prime}\right) \mathbf{M}\right]=[\mathbf{K}: \operatorname{cov}(\boldsymbol{\eta}, \mathbf{y}) \mathbf{M}] .
$$


It is essential to observe that in (e) it is necessary to assume that $\mathbf{K} \boldsymbol{\beta}$ is estimable, i.e., there exists a matrix $\mathbf{C}$ such that $\mathbf{K}=\mathbf{C X}$. As pointed out by one of the referees, this approach provides a universal engine to produce the statements (a)-(e) by choosing appropriate $\mathbf{C}$ and $\mathbf{L}$.

Thus we have, for example,

$$
\begin{aligned}
\operatorname{BLUP}(\mathbf{X} \boldsymbol{\beta}+\mathbf{Z u} \mid \mathscr{L}) & =\operatorname{BLUE}(\mathbf{X} \boldsymbol{\beta} \mid \mathscr{L})+\operatorname{BLUP}(\mathbf{Z u} \mid \mathscr{L}) \\
& =\operatorname{BLUE}(\mathbf{X} \boldsymbol{\beta} \mid \mathscr{L})+\mathbf{Z} \operatorname{BLUP}(\mathbf{u} \mid \mathscr{L}),
\end{aligned}
$$

and we have the following representations for the BLUP of $\mathbf{g}=\mathbf{X} \boldsymbol{\beta}+\mathbf{Z u}$ :

$$
\begin{aligned}
\operatorname{BLUP}(\mathbf{g}) & =\mathbf{T} \mathbf{y}+\mathbf{Z}\left(\mathbf{D} \mathbf{Z}^{\prime}+\mathbf{S}^{\prime}\right) \mathbf{W}^{-}\left(\mathbf{I}_{n}-\mathbf{T}\right) \mathbf{y} \\
& =\mathbf{T} \mathbf{y}+\mathbf{Z}\left(\mathbf{D} \mathbf{Z}^{\prime}+\mathbf{S}^{\prime}\right) \mathbf{\Sigma}^{-}\left(\mathbf{I}_{n}-\mathbf{T}\right) \mathbf{y} \\
& =\mathbf{T} \mathbf{y}+\mathbf{Z}\left(\mathbf{D} \mathbf{Z}^{\prime}+\mathbf{S}^{\prime}\right) \mathbf{M}(\mathbf{M} \boldsymbol{\Sigma} \mathbf{M})^{-} \mathbf{M} \mathbf{y}
\end{aligned}
$$

where $\mathbf{T}=\mathbf{X}\left(\mathbf{X}^{\prime} \mathbf{W}^{-} \mathbf{X}\right)^{-} \mathbf{X}^{\prime} \mathbf{W}^{-}$and $\mathbf{W} \in \mathcal{W}$,

$$
\mathcal{W}=\left\{\mathbf{W} \in \mathbb{R}^{n \times n}: \mathbf{W}=\boldsymbol{\Sigma}+\mathbf{X} \mathbf{U U}^{\prime} \mathbf{X}^{\prime}, \mathscr{C}(\mathbf{W})=\mathscr{C}(\mathbf{X}: \boldsymbol{\Sigma})\right\} .
$$

Now Fy is BLUP-sufficient for $\mathbf{g}=\mathbf{X} \boldsymbol{\beta}+\mathbf{Z u}$ under $\mathscr{L}$ in the spirit of Definition 2 if and only if there exists a matrix $\mathbf{A}$ such that

$$
\mathbf{A F}(\mathbf{X}: \mathbf{\Sigma M})=\left[\mathbf{X}: \mathbf{Z}\left(\mathbf{D Z} \mathbf{Z}^{\prime}+\mathbf{S}^{\prime}\right) \mathbf{M}\right]
$$

i.e.,

$$
\mathscr{C}\left(\begin{array}{c}
\mathbf{X}^{\prime} \\
\mathbf{M}(\mathbf{Z D}+\mathbf{S}) \mathbf{Z}^{\prime}
\end{array}\right) \subset \mathscr{C}\left(\begin{array}{c}
\mathbf{X}^{\prime} \mathbf{F}^{\prime} \\
\mathbf{M} \mathbf{\Sigma} \mathbf{F}^{\prime}
\end{array}\right) .
$$

Moreover, Fy is BLUE-sufficient for $\mathbf{X} \boldsymbol{\beta}$ if and only if

$$
\mathscr{C}\left(\begin{array}{c}
\mathbf{X}^{\prime} \\
\mathbf{0}
\end{array}\right) \subset \mathscr{C}\left(\begin{array}{c}
\mathbf{X}^{\prime} \mathbf{F}^{\prime} \\
\mathbf{M} \boldsymbol{\Sigma} \mathbf{F}^{\prime}
\end{array}\right)
$$

and $\mathbf{F y}$ is BLUP-sufficient for $\mathbf{Z u}$ if and only if

$$
\mathscr{C}\left(\begin{array}{c}
\mathbf{0} \\
\mathbf{M}(\mathbf{Z D}+\mathbf{S}) \mathbf{Z}^{\prime}
\end{array}\right) \subset \mathscr{C}\left(\begin{array}{c}
\mathbf{X}^{\prime} \mathbf{F}^{\prime} \\
\mathbf{M} \boldsymbol{\Sigma} \mathbf{F}^{\prime}
\end{array}\right) .
$$

The following theorem can now be proved along the same lines as Theorem 3.4 in Isotalo et al. (2017). We omit the proof.

Theorem 1 Consider the mixed model $\mathscr{L}=\{\mathbf{y}, \mathbf{X} \boldsymbol{\beta}+\mathbf{Z u}, \mathbf{D}, \mathbf{R}, \mathbf{S}\}$, and the following statements:

(a) $\mathbf{F y} \in \mathcal{S}(\mathbf{X} \boldsymbol{\beta})$

(b) $\mathbf{F y} \in \mathcal{S}(\mathbf{Z u})$,

(c) $\mathbf{F} \mathbf{y} \in \mathcal{S}(\mathbf{X} \boldsymbol{\beta}+\mathbf{Z u})$.

Then any of the two conditions above imply the third one. Moreover, if

$$
\mathscr{C}(\mathbf{X}) \cap \mathscr{C}\left[\mathbf{Z}\left(\mathbf{D Z} \mathbf{Z}^{\prime}+\mathbf{S}^{\prime}\right) \mathbf{M}\right]=\{\mathbf{0}\},
$$

then

$$
\text { (c) } \Longrightarrow \text { (a) and (b) . }
$$


Liu et al. (2008, p. 1511) have a slightly different definition for the linear sufficiency. According to them, the statistic Fy is BLUP-sufficient if for all predictable parametric functions $\boldsymbol{\eta}=\mathbf{K} \boldsymbol{\beta}+\mathbf{L u}$ there exists a matrix $\mathbf{A}$ such that $\mathbf{A F y}$ is the BLUP for $\boldsymbol{\eta}$ in the original model. Since $\boldsymbol{\eta}=\mathbf{K} \boldsymbol{\beta}+\mathbf{L u}$ is predictable if and only if $\mathbf{K}=\mathbf{J X}$ for some matrix $\mathbf{J}$ (while $\mathbf{L}$ can be any conformable matrix) we can re-express this definition as follows.

Definition 3 The statistic Fy is BLUP-sufficient under the model $\mathscr{L}$ if for all $\mathbf{J}$ and $\mathbf{L}$, there exists a matrix $\mathbf{A}$ such that $\mathbf{A F y}$ is the BLUP for $\boldsymbol{\eta}=$ $\mathbf{J X} \boldsymbol{\beta}+\mathbf{L u}$, and then we denote $\mathbf{F y} \in \mathcal{S}_{0}(\boldsymbol{\beta}, \mathbf{u})$.

We see that the difference between Definitions2 and 3 is that in Definition2 our object of estimation/prediction is a given predictable combination of fixed parameters and random effect like $\mathbf{g}=\mathbf{X} \boldsymbol{\beta}+\mathbf{Z u}$ (where $\mathbf{X}$ and $\mathbf{Z}$ are given and fixed) while in Definition 3 we consider all predictable combinations of the type $\boldsymbol{\eta}=\mathbf{K} \boldsymbol{\beta}+\mathbf{L u}$. Actually Kala \& Pordzik (2009, p. 635) used the linear sufficiency concept in the spirit of Definition 3 when saying that a statistic Fy is linearly sufficient if it is linearly sufficient for all estimable parametric functions of the model.

Theorem 2 Consider the mixed model $\mathscr{L}=\{\mathbf{y}, \mathbf{X} \boldsymbol{\beta}+\mathbf{Z u}, \mathbf{D}, \mathbf{R}, \mathbf{S}\}$, and denote

$$
\boldsymbol{\Sigma}=\operatorname{cov}(\mathbf{y})=\mathbf{Z D Z} \mathbf{Z}^{\prime}+\mathbf{R}+\mathbf{Z S}^{\prime}+\mathbf{S Z}^{\prime}
$$

and let $\mathbf{W} \in \mathcal{W}$, where the class $\mathcal{W}$ of matrices is defined as in $(2.13)$. Then the following statements are equivalent:

(a) $\mathbf{F y} \in \mathcal{S}(\mathbf{X} \boldsymbol{\beta}) \cap \mathcal{S}(\mathbf{u})$, i.e., $\mathbf{F y}$ is linearly sufficient for $\mathbf{X} \boldsymbol{\beta}$ and for $\mathbf{u}$.

(b) $\mathbf{F y} \in \mathcal{S}_{0}(\boldsymbol{\beta}, \mathbf{u})$, i.e., $\mathbf{F y}$ is linearly sufficient for every predictable $\mathbf{K} \boldsymbol{\beta}+\mathbf{L u}$.

(c) $\mathscr{C}\left(\begin{array}{cc}\mathbf{X}^{\prime} & \mathbf{0} \\ \mathbf{0} & \mathbf{M}(\mathbf{Z D}+\mathbf{S})\end{array}\right) \subset \mathscr{C}\left(\begin{array}{c}\mathbf{X}^{\prime} \mathbf{F}^{\prime} \\ \mathbf{M} \boldsymbol{\Sigma} \mathbf{F}^{\prime}\end{array}\right)$.

(d) $\mathscr{C}(\mathbf{X}) \subset \mathscr{C}\left(\mathbf{W F}^{\prime}\right)$ and $\mathscr{C}[\mathbf{M}(\mathbf{Z D}+\mathbf{S})] \subset \mathscr{C}\left(\mathbf{M W} \mathbf{F}^{\prime}\right)$.

(e) $\mathscr{C}(\mathbf{X}: \mathbf{Z D}+\mathbf{S}) \subset \mathscr{C}\left(\mathbf{W F}^{\prime}\right)$.

Proof Consider the statement (b). In the sense of Definition 3 , the statistic Fy is linearly sufficient, i.e., $\mathbf{F y} \in \mathcal{S}_{0}(\boldsymbol{\beta}, \mathbf{u})$, if and only if for every $\mathbf{J}$ and $\mathbf{L}$ there exists a matrix $\mathbf{A}$ such that

$$
\mathbf{A F}(\mathbf{X}: \mathbf{\Sigma} \mathbf{M})=\left[\mathbf{J X}: \mathbf{L}\left(\mathbf{D Z} \mathbf{Z}^{\prime}+\mathbf{S}^{\prime}\right) \mathbf{M}\right],
$$

which happens if and only if

$$
\mathscr{C}\left[\left(\begin{array}{cc}
\mathbf{X}^{\prime} & \mathbf{0} \\
\mathbf{0} & \mathbf{M}(\mathbf{Z D}+\mathbf{S})
\end{array}\right)\left(\begin{array}{l}
\mathbf{J}^{\prime} \\
\mathbf{L}^{\prime}
\end{array}\right)\right] \subset \mathscr{C}\left(\begin{array}{c}
\mathbf{X}^{\prime} \mathbf{F}^{\prime} \\
\mathbf{M} \boldsymbol{\Sigma} \mathbf{F}^{\prime}
\end{array}\right) .
$$

Requesting 2.22 to hold for every $\mathbf{J}$ and $\mathbf{L}$, we get

$$
\mathscr{C}\left(\begin{array}{cc}
\mathbf{X}^{\prime} & \mathbf{0} \\
\mathbf{0} & \mathbf{M}(\mathbf{Z D}+\mathbf{S})
\end{array}\right) \subset \mathscr{C}\left(\begin{array}{c}
\mathbf{X}^{\prime} \mathbf{F}^{\prime} \\
\mathbf{M} \boldsymbol{\Sigma} \mathbf{F}^{\prime}
\end{array}\right) .
$$

Thus we have shown the equivalence of (b) and (c). 
Notice that 2.23 is equivalent to

$$
\mathscr{C}\left(\begin{array}{c}
\mathbf{X}^{\prime} \\
\mathbf{0}
\end{array}\right) \subset \mathscr{C}\left(\begin{array}{c}
\mathbf{X}^{\prime} \mathbf{F}^{\prime} \\
\mathbf{M} \boldsymbol{\Sigma} \mathbf{F}^{\prime}
\end{array}\right), \quad \mathscr{C}\left(\begin{array}{c}
\mathbf{0} \\
\mathbf{M}(\mathbf{Z D}+\mathbf{S})
\end{array}\right) \subset \mathscr{C}\left(\begin{array}{c}
\mathbf{X}^{\prime} \mathbf{F}^{\prime} \\
\mathbf{M} \mathbf{\Sigma} \mathbf{F}^{\prime}
\end{array}\right) .
$$

In view of part (a) of Lemma 3 , the first inclusion in 2.24 is a condition for $\mathbf{F y} \in \mathcal{S}(\mathbf{X} \boldsymbol{\beta})$ while the second one is a condition for $\mathbf{F y} \in \mathcal{S}(\mathbf{u})$, which together mean that

$$
\mathbf{F y} \in \mathcal{S}(\mathbf{X} \boldsymbol{\beta}) \cap \mathcal{S}(\mathbf{u})
$$

This confirms the equivalence of (a) and (b). In light of part (b) of Lemma 3 , we can rewrite the first inclusion of $(2.24)$ as

$$
\mathscr{C}(\mathbf{X}) \subset \mathscr{C}\left(\mathbf{W} \mathbf{F}^{\prime}\right)
$$

while the second part of 2.24 is equivalent to

$$
\mathscr{C}[\mathbf{M}(\mathbf{Z D}+\mathbf{S})] \subset \mathscr{C}\left(\mathbf{M} \boldsymbol{\Sigma} \mathbf{F}^{\prime} \mathbf{Q}_{\mathbf{F X}}\right)=\mathscr{C}\left(\mathbf{M W} \mathbf{F}^{\prime} \mathbf{Q}_{\mathbf{F X}}\right)
$$

Using the rank rule of the matrix product, see Marsaglia \& Styan (1974, Cor. 6.2), and the disjointness condition (c) of Lemma 3 , we observe that if $\mathscr{C}(\mathbf{X}) \subset \mathscr{C}\left(\mathbf{W F}^{\prime}\right)$, then

$$
\mathscr{C}\left(\mathbf{M W F} \mathbf{Q}_{\mathbf{F X}}\right)=\mathscr{C}\left(\mathbf{M W F ^ { \prime }}\right)
$$

Thus, supposing that 2.26 holds, the inclusion 2.27 is equivalent to

$$
\mathscr{C}[\mathbf{M}(\mathbf{Z D}+\mathbf{S})] \subset \mathscr{C}\left(\mathbf{M W} \mathbf{F}^{\prime}\right) .
$$

Thereby we have shown the equivalence between (c) and (d). Now 2.29 holds if and only if $\mathbf{M}(\mathbf{Z D}+\mathbf{S})=\mathbf{M} \mathbf{W} \mathbf{F}^{\prime} \mathbf{N}_{1}$ for some $\mathbf{N}_{1}$, i.e.,

$$
\mathbf{Z D}+\mathbf{S}=\mathbf{W F}^{\prime} \mathbf{N}_{1}+\mathbf{X N}_{2}
$$

for some $\mathbf{N}_{2}$. Assuming that 2.26 holds, 2.30 implies that

$$
\mathscr{C}(\mathbf{Z D}+\mathbf{S}) \subset \mathscr{C}\left(\mathbf{W} \mathbf{F}^{\prime}\right)
$$

Thus we have proved that (d) implies (e):

$$
\mathscr{C}(\mathbf{X}: \mathbf{Z D}+\mathbf{S}) \subset \mathscr{C}\left(\mathbf{W F}^{\prime}\right) .
$$

The reverse relation, that is, $(\mathrm{e}) \Longrightarrow(\mathrm{d})$ is easily concluded.

The equivalence between (b), (c) and (e) in Theorem 2 is proved by Liu et al. (2008, §3) using different approach. 


\section{BLUPs under two different mixed models}

Consider two mixed models with the same $\mathbf{X}$ and $\mathbf{Z}$ but different overall structures

$$
\mathscr{L}_{i}=\left\{\mathbf{y}, \mathbf{X} \boldsymbol{\beta}+\mathbf{Z u}, \mathbf{D}_{i}, \mathbf{R}_{i}, \mathbf{S}_{i}\right\}, \quad i=1,2,
$$

and denote

$$
\begin{array}{r}
\operatorname{cov}\left(\begin{array}{l}
\mathbf{y} \\
\mathbf{u}
\end{array}\right)=\left(\begin{array}{cc}
\boldsymbol{\Sigma}_{i} & \mathbf{Z D}_{i}+\mathbf{S}_{i} \\
\mathbf{D}_{i} \mathbf{Z}^{\prime}+\mathbf{S}_{i}^{\prime} & \mathbf{D}_{i}
\end{array}\right)=\left(\begin{array}{cc}
\boldsymbol{\Sigma}_{i} & \mathbf{C}_{i} \\
\mathbf{C}_{i}^{\prime} & \mathbf{D}_{i}
\end{array}\right), \\
\mathbf{A}_{i}=\left(\begin{array}{c}
\boldsymbol{\Sigma}_{i} \mathbf{M} \\
\mathbf{Z} \mathbf{C}_{i} \mathbf{M}
\end{array}\right), \quad \mathbf{X}_{x}=\left(\begin{array}{c}
\mathbf{X} \\
\mathbf{X}
\end{array}\right), \quad \mathbf{X}_{0}=\left(\begin{array}{c}
\mathbf{X} \\
\mathbf{0}
\end{array}\right), \\
\mathcal{W}_{i}=\left\{\mathbf{W}_{i} \in \mathbb{R}^{n \times n}: \mathbf{W}_{i}=\boldsymbol{\Sigma}_{i}+\mathbf{X} \mathbf{U}^{\prime} \mathbf{X}^{\prime}, \mathscr{C}\left(\mathbf{W}_{i}\right)=\mathscr{C}\left(\mathbf{X}: \boldsymbol{\Sigma}_{i}\right)\right\},
\end{array}
$$

where $\boldsymbol{\Sigma}_{i}=\mathbf{Z D}_{i} \mathbf{Z}^{\prime}+\mathbf{R}_{i}+\mathbf{Z S}_{i}^{\prime}+\mathbf{S}_{i} \mathbf{Z}^{\prime}$

In this section we focus on the questions, like for example, what is the necessary and sufficient condition that every representation of the BLUE of $\mathbf{X} \boldsymbol{\beta}$ under $\mathscr{L}_{1}$ remains BLUE under $\mathscr{L}_{2}$, for which relation we will use the short notation

$$
\mathcal{B}\left(\mathbf{X} \boldsymbol{\beta} \mid \mathscr{L}_{1}\right) \subset \mathcal{B}\left(\mathbf{X} \boldsymbol{\beta} \mid \mathscr{L}_{2}\right)
$$

i.e.,

$$
\left\{\operatorname{BLUE}\left(\mathbf{X} \boldsymbol{\beta} \mid \mathscr{L}_{1}\right)\right\} \subset\left\{\operatorname{BLUE}\left(\mathbf{X} \boldsymbol{\beta} \mid \mathscr{L}_{2}\right)\right\}
$$

Thus we have, for example,

$$
\begin{aligned}
\mathcal{B}\left(\mathbf{X} \boldsymbol{\beta} \mid \mathscr{L}_{i}\right) & =\left\{\mathbf{A y}: \mathbf{A}\left(\mathbf{X}: \boldsymbol{\Sigma}_{i} \mathbf{M}\right)=(\mathbf{X}: \mathbf{0})\right\} \\
\mathcal{B}\left(\mathbf{Z u} \mid \mathscr{L}_{i}\right) & =\left\{\mathbf{B y}: \mathbf{B}\left(\mathbf{X}: \boldsymbol{\Sigma}_{i} \mathbf{M}\right)=\left[\mathbf{0}: \mathbf{Z}\left(\mathbf{D}_{i} \mathbf{Z}^{\prime}+\mathbf{S}_{i}^{\prime}\right) \mathbf{M}\right]\right\} .
\end{aligned}
$$

Recall that in this situation the corresponding comments as given on 1.17 are holding.

For the following lemma, see Haslett \& Puntanen (2010a b, 2011, 2013), Liu \& Wang (2013), and Wang \& Liu (2013).

Lemma 4 Using the above notation, consider the following statements:
(a) $\mathcal{B}\left(\mathbf{X} \boldsymbol{\beta} \mid \mathscr{L}_{1}\right) \subset \mathcal{B}\left(\mathbf{X} \boldsymbol{\beta} \mid \mathscr{L}_{2}\right)$,
(b) $\mathcal{B}\left(\mathbf{Z u} \mid \mathscr{L}_{1}\right) \subset \mathcal{B}\left(\mathbf{Z u} \mid \mathscr{L}_{2}\right)$,
(c) $\mathcal{B}\left(\mathbf{X} \boldsymbol{\beta}+\mathbf{Z u} \mid \mathscr{L}_{1}\right) \subset \mathcal{B}\left(\mathbf{X} \boldsymbol{\beta}+\mathbf{Z u} \mid \mathscr{L}_{2}\right)$
(d) $\mathcal{B}\left(\mathbf{X} \boldsymbol{\beta} \mid \mathscr{L}_{1}\right) \subset \mathcal{B}\left(\mathbf{X} \boldsymbol{\beta} \mid \mathscr{L}_{2}\right)$ and $\mathcal{B}\left(\mathbf{Z u} \mid \mathscr{L}_{1}\right) \subset \mathcal{B}\left(\mathbf{Z u} \mid \mathscr{L}_{2}\right)$,
$\left(\mathrm{a}_{*}\right) \mathscr{C}\left(\boldsymbol{\Sigma}_{2} \mathbf{M}\right) \subset \mathscr{C}\left(\boldsymbol{\Sigma}_{1} \mathbf{M}\right)$,
$\left(\mathrm{b}_{*}\right) \mathscr{C}\left(\begin{array}{c}\boldsymbol{\Sigma}_{2} \mathbf{M} \\ \mathbf{Z C}_{2} \mathbf{M}\end{array}\right) \subset \mathscr{C}\left(\begin{array}{cc}\mathbf{X} & \boldsymbol{\Sigma}_{1} \mathbf{M} \\ \mathbf{0} & \mathbf{Z C}_{1} \mathbf{M}\end{array}\right)$
$\left(c_{*}\right) \mathscr{C}\left(\begin{array}{c}\boldsymbol{\Sigma}_{2} \mathbf{M} \\ \mathbf{Z} \mathbf{C}_{2} \mathbf{M}\end{array}\right) \subset \mathscr{C}\left(\begin{array}{cc}\mathbf{X} & \boldsymbol{\Sigma}_{1} \mathbf{M} \\ \mathbf{X} & \mathbf{Z C}_{1} \mathbf{M}\end{array}\right)$,
$\left(\mathrm{d}_{*}\right) \mathscr{C}\left(\begin{array}{c}\boldsymbol{\Sigma}_{2} \mathbf{M} \\ \mathbf{Z} \mathbf{C}_{2} \mathbf{M}\end{array}\right) \subset \mathscr{C}\left(\begin{array}{c}\boldsymbol{\Sigma}_{1} \mathbf{M} \\ \mathbf{Z} \mathbf{C}_{1} \mathbf{M}\end{array}\right)$. 
Then (a) $\Longleftrightarrow\left(\mathrm{a}_{*}\right), \ldots,(\mathrm{d}) \Longleftrightarrow\left(\mathrm{d}_{*}\right)$.

Next we prove some interesting relations between the statements of the above Lemma.

Theorem 3 Using the notation of the Lemma 不, the following holds:

(i) (a) \& (b) $\Longrightarrow$ (c), i.e., (d) $\Longrightarrow$ (c).

(ii) (a) \& (c) $\Longrightarrow$ (b).

(iii) (b) \& (c) $\Longrightarrow$ (a) if

$$
\mathscr{C}(\mathbf{X}) \cap \mathscr{C}\left(\mathbf{Z C}_{1} \mathbf{M}\right)=\{\mathbf{0}\} .
$$

(iv) (c) $\Longrightarrow$ (d) if and only if

$$
\mathbf{X}_{x}^{\prime} \mathbf{P}_{\mathbf{A}_{1}} \mathbf{A}_{2}=\mathbf{X}_{x}^{\prime} \mathbf{A}_{2},
$$

where the matrix $\mathbf{X}_{x}^{\prime}$ can be cancelled if $\mathscr{C}\left(\mathbf{A}_{1}\right) \cap \mathscr{C}\left(\mathbf{X}_{x}\right)^{\perp}=\{\mathbf{0}\}$.

Proof Clearly the inclusion (d) implies (c) but the reverse relation holds only in special cases as stated by Haslett \& Puntanen (2013, p. 37). Claim (c) is equivalent to $\mathbf{P}_{\left(\mathbf{X}_{x}: \mathbf{A}_{1}\right)} \mathbf{A}_{2}=\mathbf{A}_{2}$, which can be written as

$$
\mathbf{P}_{\mathbf{A}_{1}} \mathbf{A}_{2}+\mathbf{P}_{\mathbf{Q}_{\mathbf{A}_{1}} \mathbf{X}_{x}} \mathbf{A}_{2}=\mathbf{A}_{2} \text {. }
$$

Thus, in this situation (d) holds if and only if $\mathbf{P}_{\mathbf{Q}_{\mathbf{A}_{1}}} \mathbf{x}_{x} \mathbf{A}_{2}=\mathbf{0}$, i.e.,

$$
\mathbf{X}_{x}^{\prime} \mathbf{Q}_{\mathbf{A}_{1}} \mathbf{A}_{2}=\mathbf{0},
$$

or, equivalently,

$$
\mathbf{X}_{x}^{\prime} \mathbf{P}_{\mathbf{A}_{1}} \mathbf{A}_{2}=\mathbf{X}_{x}^{\prime} \mathbf{A}_{2} .
$$

Trivially (3.13) holds if

$$
\mathscr{C}\left(\mathbf{A}_{2}\right) \subset \mathscr{C}\left(\mathbf{A}_{1}\right)
$$

but (3.14) is not a necessary condition. It is necessary if we can cancel $\mathbf{X}_{x}^{\prime}$ from (3.12) and this can be done, on account of Marsaglia \& Styan (1974, Th. 2), if

$$
\operatorname{rank}\left(\mathbf{X}_{x}^{\prime} \mathbf{Q}_{\mathbf{A}_{1}}\right)=\operatorname{rank}\left(\mathbf{Q}_{\mathbf{A}_{1}}\right),
$$

i.e.,

$$
\begin{aligned}
\operatorname{rank}\left(\mathbf{X}_{x}^{\prime} \mathbf{Q}_{\mathbf{A}_{1}}\right) & =\operatorname{rank}\left(\mathbf{Q}_{\mathbf{A}_{1}}\right)-\operatorname{dim} \mathscr{C}\left(\mathbf{Q}_{\mathbf{A}_{1}}\right)^{\perp} \cap \mathscr{C}\left(\mathbf{X}_{x}\right)^{\perp} \\
& =\operatorname{rank}\left(\mathbf{Q}_{\mathbf{A}_{1}}\right),
\end{aligned}
$$

which happens if and only if $\mathscr{C}\left(\mathbf{A}_{1}\right) \cap \mathscr{C}\left(\mathbf{X}_{x}\right)^{\perp}=\{\mathbf{0}\}$. Thus we have proved (iv).

To consider claim (ii), we note that the statements (a) and (c) imply that

$$
\begin{aligned}
\boldsymbol{\Sigma}_{2} \mathbf{M} & =\boldsymbol{\Sigma}_{1} \mathbf{M K}_{1}, \\
\boldsymbol{\Sigma}_{2} \mathbf{M} & =\mathbf{X L}_{1}+\boldsymbol{\Sigma}_{1} \mathbf{M L}_{2}, \\
\mathbf{Z C}_{2} \mathbf{M} & =\mathbf{X L}_{1}+\mathbf{Z C}_{1} \mathbf{M L}_{2},
\end{aligned}
$$


for some matrices $\mathbf{K}_{1}, \mathbf{L}_{1}$, and $\mathbf{L}_{2}$. The first two equations above imply that

$$
\mathbf{X L}_{1}=\boldsymbol{\Sigma}_{1} \mathbf{M}\left(\mathbf{K}_{1}-\mathbf{L}_{2}\right) .
$$

In view of $\mathscr{C}(\mathbf{X}) \cap \mathscr{C}\left(\boldsymbol{\Sigma}_{1} \mathbf{M}\right)=\{\mathbf{0}\},(3.18)$ implies that $\mathbf{X L}_{1}=\mathbf{0}$. Substituting this into $3.17 \mathrm{~b})$ and $3.17 \mathrm{c}$ confirms the claim (ii).

To prove (iii), notice that (b) and (c) imply the existence of matrices $\mathbf{K}_{i}, \mathbf{L}_{i}, i=1,2$, such that

$$
\begin{aligned}
& \boldsymbol{\Sigma}_{2} \mathbf{M}=\mathbf{X K}_{1}+\boldsymbol{\Sigma}_{1} \mathbf{M K}_{2}, \quad \mathbf{Z C}_{2} \mathbf{M}=\mathbf{Z C}_{1} \mathbf{M K}_{2}, \\
& \boldsymbol{\Sigma}_{2} \mathbf{M}=\mathbf{X L}_{1}+\boldsymbol{\Sigma}_{1} \mathbf{M L}_{2}, \quad \mathbf{Z C}_{2} \mathbf{M}=\mathbf{X L}_{1}+\mathbf{Z C}_{1} \mathbf{M L}_{2} \text {. }
\end{aligned}
$$

The right-hand side equations above imply that

$$
\mathbf{X L}_{1}=\mathbf{Z C}_{1} \mathbf{M}\left(\mathbf{K}_{2}-\mathbf{L}_{2}\right) \text {. }
$$

If the disjointness $\mathscr{C}(\mathbf{X}) \cap \mathscr{C}\left(\mathbf{Z} \mathbf{C}_{1} \mathbf{M}\right)=\{\mathbf{0}\}$, holds then 3.20 implies that $\mathbf{X L}_{1}=\mathbf{0}$ which would further imply (a). Thus we have proved our claim (iii).

Rong \& Liu (2010) consider the predictable parametric function $\boldsymbol{\eta}=\mathbf{K} \boldsymbol{\beta}+$ $\mathbf{L u}=\mathbf{J X} \boldsymbol{\beta}+\mathbf{L u}$ and its BLUP which has a representation

$$
\begin{aligned}
\operatorname{BLUP}\left(\boldsymbol{\eta} \mid \mathscr{L}_{1}\right) & =\tilde{\boldsymbol{\eta}}_{1} \\
& =\mathbf{J} \mathbf{T}_{1} \mathbf{y}+\mathbf{L C}_{1}^{\prime} \mathbf{W}_{1}^{-}\left(\mathbf{I}_{n}-\mathbf{T}_{1}\right) \mathbf{y} \\
& =\mathbf{J} \mathbf{T}_{1} \mathbf{y}+\mathbf{L} \mathbf{G}_{1} \mathbf{y}
\end{aligned}
$$

where

$$
\mathbf{T}_{1}=\mathbf{X}\left(\mathbf{X}^{\prime} \mathbf{W}_{1}^{-} \mathbf{X}\right)^{-} \mathbf{X}^{\prime} \mathbf{W}_{1}^{-}, \quad \mathbf{G}_{1}=\mathbf{C}_{1}^{\prime} \mathbf{W}_{1}^{-}\left(\mathbf{I}_{n}-\mathbf{T}_{1}\right)
$$

and $\mathbf{W}_{1}$ is any member of the class $\mathcal{W}_{1}$ defined in (3.4). Rong \& Liu (2010 Th. 1) investigate the conditions under which $\tilde{\boldsymbol{\eta}}_{1}$ continues to be BLUP under the model $\mathscr{L}_{2}$. This problem is rather similar to claim (c) of Lemma 4 , dealing with the inclusion

$$
\mathcal{B}\left(\mathbf{X} \boldsymbol{\beta}+\mathbf{Z u} \mid \mathscr{L}_{1}\right) \subset \mathcal{B}\left(\mathbf{X} \boldsymbol{\beta}+\mathbf{Z u} \mid \mathscr{L}_{2}\right)
$$

However, there is one crucial difference between Lemma 4 and the approach in Rong \& Liu (2010, Th. 1): in Lemma 4 the object of prediction/estimation is fixed, while Rong \& Liu (2010) consider the set of all predictable parametric functions $\boldsymbol{\eta}=\mathbf{K} \boldsymbol{\beta}+\mathbf{L u}=\mathbf{J X} \boldsymbol{\beta}+\mathbf{L u}$, that is, $\mathbf{J}$ and $\mathbf{L}$ are free to vary. They request $\tilde{\boldsymbol{\eta}}_{1}$ to be BLUP for all (conformable) matrices $\mathbf{J}$ and $\mathbf{L}$ :

$$
\left(\mathbf{J T}_{1}+\mathbf{L G}_{1}\right)\left(\mathbf{X}: \boldsymbol{\Sigma}_{2} \mathbf{M}\right)=\left(\mathbf{J X}: \mathbf{L C}_{2}^{\prime} \mathbf{M}\right)
$$

i.e.,

$$
\left(\mathbf{J T}_{1}+\mathbf{L G}_{1}\right) \boldsymbol{\Sigma}_{2} \mathbf{M}=\mathbf{L C}_{2}^{\prime} \mathbf{M}
$$


Equation 3.25 holds for every $\mathbf{J}$ and $\mathbf{L}$ if and only if $\mathbf{T}_{1} \boldsymbol{\Sigma}_{2} \mathbf{M}=\mathbf{0}$ and $\mathbf{G}_{1} \Sigma_{2} \mathbf{M}=\mathbf{C}_{2}^{\prime} \mathbf{M}$, i.e.,

$$
\mathbf{X}^{\prime} \mathbf{W}_{1}^{-} \boldsymbol{\Sigma}_{2} \mathbf{M}=\mathbf{0}, \quad \mathbf{C}_{1}^{\prime} \mathbf{W}_{1}^{-}\left(\mathbf{I}_{n}-\mathbf{T}_{1}\right) \boldsymbol{\Sigma}_{2} \mathbf{M}=\mathbf{C}_{2}^{\prime} \mathbf{M}
$$

which are equivalent, correspondingly, to

$$
\mathbf{T}_{1} \mathbf{y} \in \mathcal{B}\left(\mathbf{X} \boldsymbol{\beta} \mid \mathscr{L}_{2}\right), \quad \mathbf{G}_{1} \mathbf{y} \in \mathcal{B}\left(\mathbf{u} \mid \mathscr{L}_{2}\right)
$$

Thus we have proved the following result.

Theorem 4 Consider the linear mixed models $\mathscr{L}_{1}$ and $\mathscr{L}_{2}$, and denote

$$
\begin{aligned}
\operatorname{BLUP}\left(\boldsymbol{\eta} \mid \mathscr{L}_{1}\right)=\tilde{\boldsymbol{\eta}}_{1} & =\mathbf{J} \mathbf{T}_{1} \mathbf{y}+\mathbf{L} \mathbf{C}_{1}^{\prime} \mathbf{W}_{1}^{-}\left(\mathbf{I}_{n}-\mathbf{T}_{1}\right) \mathbf{y} \\
& =\mathbf{J} \mathbf{T}_{1} \mathbf{y}+\mathbf{L} \mathbf{G}_{1} \mathbf{y}
\end{aligned}
$$

where $\mathbf{W}_{1}$ is any member of the class $\mathcal{W}_{1}$ defined in (3.4). Then

(a) $\tilde{\boldsymbol{\eta}}_{1} \in \mathcal{B}\left(\mathbf{J X} \boldsymbol{\beta}+\mathbf{L u} \mid \mathscr{L}_{2}\right)$ for all $\mathbf{J}$ and $\mathbf{L}$

if and only if the following two conditions hold:

(b) $\mathbf{T}_{1} \mathbf{y} \in \mathcal{B}\left(\mathbf{X} \boldsymbol{\beta} \mid \mathscr{L}_{2}\right)$,

(c) $\mathbf{G}_{1} \mathbf{y} \in \mathcal{B}\left(\mathbf{u} \mid \mathscr{L}_{2}\right)$.

Theorem 4 above and Theorem 5 below are related to Rong \& Liu (2010 Th. 1, Th. 2). Our formulation and the proofs are, however, different.

Theorem 5 Consider the linear mixed models $\mathscr{L}_{1}$ and $\mathscr{L}_{2}$, and assume that

$$
\mathscr{C}\left(\boldsymbol{\Sigma}_{2}\right) \subset \mathscr{C}\left(\mathbf{X}: \boldsymbol{\Sigma}_{1}\right)
$$

Then

(i) 3.29 and (b) of Theorem 4 together are equivalent to

$$
\mathcal{B}\left(\mathbf{X} \boldsymbol{\beta} \mid \mathscr{L}_{1}\right) \subset \mathcal{B}\left(\mathbf{X} \boldsymbol{\beta} \mid \mathscr{L}_{2}\right)
$$

which further is equivalent to

$$
\mathscr{C}\left(\boldsymbol{\Sigma}_{2} \mathbf{M}\right) \subset \mathscr{C}\left(\boldsymbol{\Sigma}_{1} \mathbf{M}\right)
$$

(ii) 3.29 and (c) of Theorem 4 together are equivalent to

$$
\mathcal{B}\left(\mathbf{u} \mid \mathscr{L}_{1}\right) \subset \mathcal{B}\left(\mathbf{u} \mid \mathscr{L}_{2}\right)
$$

which further is equivalent to

$$
\mathscr{C}\left(\begin{array}{l}
\boldsymbol{\Sigma}_{2} \mathbf{M} \\
\mathbf{C}_{2} \mathbf{M}
\end{array}\right) \subset \mathscr{C}\left(\begin{array}{cc}
\mathbf{X} & \boldsymbol{\Sigma}_{1} \mathbf{M} \\
\mathbf{0} & \mathbf{C}_{1} \mathbf{M}
\end{array}\right)
$$


Proof Regarding the condition 3.29, notice that, see, e.g., Puntanen et al. (2011, §5.1),

$$
\mathscr{C}\left(\mathbf{X}: \boldsymbol{\Sigma}_{i}\right)=\mathscr{C}\left(\mathbf{X}: \boldsymbol{\Sigma}_{i} \mathbf{M}\right)=\mathscr{C}\left(\mathbf{W}_{i}\right), \quad i=1,2
$$

Using (3.34) we observe that the following statements are equivalent:

$$
\begin{aligned}
\mathscr{C}\left(\boldsymbol{\Sigma}_{2}\right) & \subset \mathscr{C}\left(\mathbf{X}: \boldsymbol{\Sigma}_{1}\right), \\
\mathscr{C}\left(\mathbf{W}_{2}\right)=\mathscr{C}\left(\mathbf{X}: \boldsymbol{\Sigma}_{2} \mathbf{M}\right) & \subset \mathscr{C}\left(\mathbf{X}: \boldsymbol{\Sigma}_{1} \mathbf{M}\right)=\mathscr{C}\left(\mathbf{W}_{1}\right), \\
\mathscr{C}\left(\boldsymbol{\Sigma}_{2} \mathbf{M}\right) & \subset \mathscr{C}\left(\mathbf{X}: \boldsymbol{\Sigma}_{1} \mathbf{M}\right) .
\end{aligned}
$$

The general representation for the $\operatorname{BLUE}\left(\mathbf{X} \boldsymbol{\beta} \mid \mathscr{L}_{1}\right)$ is $\mathbf{T}_{0} \mathbf{y}$, where

$$
\mathbf{T}_{0}=\mathbf{T}_{1}+\mathbf{N}_{1} \mathbf{Q}_{\mathbf{W}_{1}}
$$

with $\mathbf{N}_{1}$ being free to vary. In view of $\left[3.35\right.$, we can conclude that $\mathbf{T}_{0} \mathbf{y}$ is the BLUE for $\mathbf{X} \boldsymbol{\beta}$ for any matrix $\mathbf{N}_{1}$, which means that every representation of BLUE for $\mathbf{X} \boldsymbol{\beta}$ under $\mathscr{L}_{1}$ remains BLUE under $\mathscr{L}_{2}$, i.e.,

$$
\mathcal{B}\left(\mathbf{X} \boldsymbol{\beta} \mid \mathscr{L}_{1}\right) \subset \mathcal{B}\left(\mathbf{X} \boldsymbol{\beta} \mid \mathscr{L}_{2}\right)
$$

which happens if and only if $\mathscr{C}\left(\boldsymbol{\Sigma}_{2} \mathbf{M}\right) \subset \mathscr{C}\left(\boldsymbol{\Sigma}_{1} \mathbf{M}\right)$.

In the corresponding way, the general representation for the $\operatorname{BLUP}(\mathbf{u})$ un$\operatorname{der} \mathscr{L}_{1}$ is $\mathbf{G}_{0} \mathbf{y}$, where

$$
\mathbf{G}_{0}=\mathbf{G}_{1}+\mathbf{N}_{2} \mathbf{Q}_{\mathbf{W}_{1}}
$$

where $\mathbf{N}_{2}$ is free to vary. Thereby, in view of (3.35), we can conclude that (ii) holds. We notice in passing that substituting

$$
\Sigma_{2} \mathbf{M}=\mathbf{X B}_{1}+\Sigma_{1} \mathbf{M B}_{2}
$$

into $\mathbf{C}_{1}^{\prime} \mathbf{W}_{1}^{-}\left(\mathbf{I}_{n}-\mathbf{T}_{1}\right) \boldsymbol{\Sigma}_{2} \mathbf{M}=\mathbf{C}_{2}^{\prime} \mathbf{M}$, which is the condition for $\mathbf{G}_{1} \mathbf{y}$ being a $\operatorname{BLUP}(\mathbf{u})$ under $\mathscr{L}_{2}$, yields

$$
\mathrm{C}_{1}^{\prime} \mathrm{MB}_{2}=\mathrm{C}_{2}^{\prime} \mathbf{M}
$$

Combining 3.39 and 3.40 gives

$$
\left(\begin{array}{l}
\boldsymbol{\Sigma}_{2} \mathbf{M} \\
\mathbf{C}_{2}^{\prime} \mathbf{M}
\end{array}\right)=\left(\begin{array}{cc}
\mathbf{X} & \boldsymbol{\Sigma}_{1} \mathbf{M} \\
\mathbf{0} & \mathbf{C}_{1}^{\prime} \mathbf{M}
\end{array}\right)\left(\begin{array}{l}
\mathbf{B}_{1} \\
\mathbf{B}_{2}
\end{array}\right)
$$

which is just the condition for $\mathcal{B}\left(\mathbf{u} \mid \mathscr{L}_{1}\right) \subset \mathcal{B}\left(\mathbf{u} \mid \mathscr{L}_{2}\right)$. 


\section{Concluding remarks}

In this paper we have considered the linear sufficiency of $\mathbf{F y}$ for $\mathbf{X} \boldsymbol{\beta}$, for $\mathbf{Z u}$ and for $\mathbf{X} \boldsymbol{\beta}+\mathbf{Z u}$, when dealing with the linear mixed model $\mathbf{y}=\mathbf{X} \boldsymbol{\beta}+\mathbf{Z u}+\mathbf{e}$. The conditions for these properties are documented in the literature but not so much their mutual relations as we have done in Theorems 1 and 2 , Liu et al. (2008) introduced a slightly different definition for the linear sufficiency and that inspired particularly the Theorem 2

We have also considered the conditions under which the BLUEs and/or BLUPs under one mixed model continue to be BLUEs and/or BLUPs under the other mixed model. These conditions appear as well in literature but our main contributions concern the mutual relations of the conditions, as presented in Theorem 3. These problems were approached differently by Rong \& Liu (2010). In Theorems 4 and 5 we have shown how their results are related to those obtained by our approach.

This link between BLUP and REML is outlined by Harville (1977) for the general linear model using the Normal distribution. The linear sufficiency results given in our paper can be applied to REML estimates, with the proviso that in REML covariance matrices are not treated as known but are estimated from the available data in an iterative procedure that at each step also reestimates $\boldsymbol{\beta}$ and $\mathbf{u}$. At convergence, the REML estimates are estimated best linear unbiased estimates (sometimes called EBLUP). The linear sufficiency results in our paper can then be applied to mixed linear models estimated using REML, but applying them using estimated covariances instead of $\mathbf{D}$ and $\mathbf{R}$ and $\mathbf{S}$ of 1.5 . Note too that in most applications of REML, $\mathbf{D}$ and $\mathbf{R}$ are highly structured and $\mathbf{S}=\mathbf{0}$, because otherwise there can be more parameters to estimate in $\boldsymbol{\beta}, \mathbf{u}, \mathbf{D}, \mathbf{R}$ and $\mathbf{S}$ than there are observations.

The matrix $\mathbf{M}=\mathbf{I}_{n}-\mathbf{P}_{\mathbf{X}}$ being orthogonal to $\mathbf{X}$ plays a key role in REML because premultiplication of 1.5 by $\mathbf{M}$ produces a reduced model from which $\mathbf{X} \boldsymbol{\beta}$ has been eliminated. Actually, $\mathbf{M y}$ is linearly sufficient for $\mathbf{Z u}$ under the mixed model (1.5), because then the condition (2.17) becomes

$$
\mathscr{C}\left[\mathbf{M}(\mathbf{Z D}+\mathbf{S}) \mathbf{Z}^{\prime}\right] \subset \mathscr{C}(\mathbf{M} \mathbf{\Sigma} \mathbf{M})=\mathscr{C}(\mathbf{M} \boldsymbol{\Sigma}),
$$

which obviously holds in view of $\mathscr{C}\left[(\mathbf{Z D}+\mathbf{S}) \mathbf{Z}^{\prime}\right] \subset \mathscr{C}(\boldsymbol{\Sigma})$. This reduced model has the same BLUPs and EBLUPs for $\mathbf{u}$ as those from 1.5 . This is why for REML, predictions of the random components in $\mathbf{u}$ do not depend on the estimates of $\mathbf{X} \boldsymbol{\beta}$. Moreover, we would like to emphasize that linear sufficiency of a given statistic Fy is a property of the model under consideration. This is the key seeing why linear BLUP-sufficiency means linear sufficiency for EBLUP.

McGilchrist (1994) first used BLUP to obtain approximate restricted maximum-likelihood (REML) estimates for generalized linear mixed models, but this topic is beyond the scope of our paper.

Acknowledgements Thanks go to the anonymous referees for constructive remarks. Part of this research was done during the meeting of a Research Group on Mixed and Multivariate 
Models in the Mathematical Research and Conference Center, Bȩdlewo, Poland, October 2016, supported by the Stefan Banach International Mathematical Center.

\section{References}

Baksalary JK, Kala R (1981) Linear transformations preserving best linear unbiased estimators in a general Gauss-Markoff model. Ann Stat 9:913-916 DOI

Baksalary JK, Kala R (1986) Linear sufficiency with respect to a given vector of parametric functions. J Stat Plan Inf 14:331-338 DOI

Christensen R (2011) Plane Answers to Complex Questions: The Theory of Linear Models. 4. Springer, New York DOI

Drygas H (1970) The Coordinate-Free Approach to Gauss-Markov Estimation. Springer, Berlin DOI

Drygas H (1983) Sufficiency and completeness in the general Gauss-Markov model. Sankhyā Ser A 45:88-98

Harville DA (1977) Maximum likelihood approaches to variance component estimation and to related problems. J Amer Statist Assoc 72:320-338 DOI

Haslett SJ, Isotalo J, Liu Y, Puntanen S (2014) Equalities between OLSE, BLUE and BLUP in the linear model. Stat Pap 55:543-561. DOI

Haslett SJ, Puntanen S (2010a) Equality of BLUEs or BLUPs under two linear models using stochastic restrictions. Stat Pap 51:465-475 DOI

Haslett SJ, Puntanen S (2010b) A note on the equality of the BLUPs for new observations under two linear models. Acta Comment Univ Tartu Math 14:27-33 www

Haslett SJ, Puntanen S (2011) On the equality of the BLUPs under two linear mixed models. Metrika 74:381-395. DOI

Haslett SJ, Puntanen S (2013) A review of conditions under which BLUEs and/or BLUPs in one linear mixed model are also BLUEs and/or BLUPs in another. Calcutta Statist Assoc Bull 65:25-41 DOI

Haslett SJ, Puntanen S, Arendacká B (2015) The link between the mixed and fixed linear models revisited. Stat Pap 56:849-861 DOI

Isotalo J, Markiewicz A, Puntanen S (2017) Some properties of linear prediction sufficiency in the linear model. Proceedings of the LINSTAT-2016 (Istanbul, Turkey, 22-25 August 2016.) Müjgan Tez and Dietrich von Rosen, Editors. Springer, in press.

Isotalo J, Puntanen S (2006) Linear prediction sufficiency for new observations in the general Gauss-Markov model. Commun Stat Theory Methods 35:1011-1023 DOI

Kala R, Markiewicz A, Puntanen S (2017) Some further remarks on the linear sufficiency in the linear model. Applied and Computational Matrix Analysis. Natália Bebiano, editor, Springer, to appear. URL

Kala R, Pordzik PR (2009) Estimation in singular partitioned, reduced or transformed linear models. Stat Pap 50:633-638 DOI

Kala R, Puntanen S, Tian Y (2017) Some notes on linear sufficiency. Stat Pap, available online. DOI

Liu XQ, Rong JY, Liu JY (2008) Best linear unbiased prediction for linear combinations in general mixed linear models. J Multivar Anal 99:1503-1517 DOI

Liu X, Wang QW (2013) Equality of the BLUPs under the mixed linear model when random components and errors are correlated. J Multivar Anal 116:297-309 DOI

Marsaglia G, Styan GPH (1974) Equalities and inequalities for ranks of matrices. Linear Multilinear Algebra 2:269-292 DOI

McGilchrist CA (1994) Estimation in generalized mixed models. J Roy Statist Soc Ser B 56:61-69 URL

Puntanen S, Styan GPH, Isotalo J (2011) Matrix Tricks for Linear Statistical Models: Our Personal Top Twenty. Springer, Heidelberg DOI

Rao CR (1973) Representations of best linear estimators in the Gauss-Markoff model with a singular dispersion matrix. J Multivar Anal 3:276-292 DOI

Rao CR, Mitra SK (1971) Generalized Inverse of Matrices and Its Applications. Wiley, New York 
Rong JY, Liu XQ (2010) On misspecification of the dispersion matrix in mixed linear models. Stat Pap 51:445-453 DOI

Tian Y, Puntanen S (2009) On the equivalence of estimations under a general linear model and its transformed models. Linear Algebra Appl 430:2622-2641 DOI

Wang QW, Liu X (2013) The equalities of BLUPs for linear combinations under two general linear mixed models. Commun Stat Theory Methods 42:3528-3543 DOI 